\title{
Variations
}

Variations

Revue internationale de théorie critique

$20 \mid 2017$

Expériences oppositionnelles

\section{Reconnaissance et dispositionnalisme}

Une discussion de la théorie de la reconnaissance d'Axel Honneth

Fabien Granjon

\section{CpenEdition}

Journals

Édition électronique

URL : http://journals.openedition.org/variations/858

DOI : 10.4000/variations.858

ISSN : 1968-3960

Éditeur

Les amis de Variations

Référence électronique

Fabien Granjon, «Reconnaissance et dispositionnalisme », Variations [En ligne], 20 | 2017, mis en ligne le 25 avril 2017, consulté le 25 février 2021. URL : http://journals.openedition.org/variations/858

DOI : https://doi.org/10.4000/variations.858

Ce document a été généré automatiquement le 25 février 2021.

Les ami•e•s de Variations 


\title{
Reconnaissance et dispositionnalisme
}

\author{
Une discussion de la théorie de la reconnaissance d'Axel Honneth
}

Fabien Granjon

1 Les débats en sciences et philosophie sociales ayant pour objet le thème de la reconnaissance sont aujourd'hui pléthoriques ${ }^{1}$. Dans le cadre de cet article, nous souhaiterions revenir plus particulièrement sur la théorie de la reconnaissance telle qu'elle est développée par Axel Honneth dans ses plus récents ouvrages ${ }^{2}$, en tant que théorie sociale normative qui a trait à la lutte des sujets pour la reconnaissance mutuelle de leur identité. Pour le philosophe allemand, la logique du rapport de reconnaissance est une confirmation des dimensions identitaires des sujets qui s'y inscrivent et «qui introduit d'emblée une tension morale dans la vie sociale ${ }^{3}$ ». La théorie de la reconnaissance appréhende ainsi les ressorts d'une éthicité, c'est-à-dire s'intéresse aux universaux normatifs d'une vie réussie recouvrant «l'ensemble des conditions intersubjectives dont on peut prouver qu'elles constituent les présupposés nécessaires de la réalisation individuelle de soi $^{4}{ } »$. Ce projet de compréhension critique des sociétés capitalistes avancées a de nombreuses fois fait la preuve de son heuristique, notamment dans le cadre de recherche traitant du travail ou encore de l'exclusion ${ }^{5}$. Nous voudrions toutefois discuter certains de ses attendus théoriques depuis une perspective que l'on pourrait désigner comme un constructivisme de type dispositionnaliste. Ce dialogue permettra d'aller plus loin dans la qualification des phénomènes de reconnaissance, de mépris et d'idéologie. Il nous semble en effet qu'un couplage de cette nature pourrait conduire à améliorer la portée à la fois descriptive et critique du système conceptuel de la théorie de la reconnaissance.

\section{De la reconnaissance}

2 La théorie de la reconnaissance s'appuie, en son cœur, sur une discussion entrelacée de la philosophie sociale consignée dans les écrits d'Iéna de Georg W. F. Hegel et de la psychologie sociale et pragmatique de George Herbert Mead («matérialiste » précise 
Honneth). La fertilisation croisée de ces apports conduit Honneth à avancer une proposition théorique rendant compte des processus de transformation sociale à l'aune d'exigences normatives structurellement comprises dans la relation de reconnaissance intersubjective. Sa théorie de la reconnaissance est ainsi construite sur une vision posthégélienne de la lutte sociale qui s'appuie fondamentalement sur trois ordres de conflits sociaux potentiels, faisant référence en amont à trois manières de développer une relation harmonieuse à soi-même. Pour Honneth, ces sphères normatives de la reconnaissance sont l'amour, le droit et l'estime sociale (l'éthicité chez Hegel) et forment l'infrastructure morale des sociétés modernes. Elles sont donc les trois sources morales et les trois espaces de lutte que les sujets investissent afin de faire reconnaître leurs besoins affectifs, leurs droits et leurs aptitudes pratiques. Cette vision tripartite correspond d'après Honneth à la «structure réelle des rapports sociaux » et, puisque fondée sur une phénoménologie des faits sociaux, peut en cela être aisément confrontée à des preuves empiriques.

3 Honneth situe le premier mode de reconnaissance mutuelle au sein de la sphère de la reproduction sociale qui relève de l'amour. Il s'agit d'une sphère d'interaction qui comprend «toutes les relations primaires qui, sur le modèle des rapports érotiques, amicaux ou familiaux impliquent des liens affectifs puissants entre un nombre restreint de personnes ${ }^{6} »$. Premier degré de la reconnaissance réciproque amenant confiance en soi, les sujets y développent des expériences de leur sollicitude et de leur attachement mutuels tachant de maintenir un équilibre entre autonomie individuelle et dépendance symbiotique. La deuxième sphère de reconnaissance est celle qui apporte une garantie intersubjective aux droits des sujets (respect de soi). Elle rend compte des obligations normatives auxquelles nous sommes tenus à l'égard d'autrui en tant qu'il est membre de la communauté et porteur des droits qui s'y rattachent. Par là même, elle permet en retour de se considérer comme étant aussi un sujet juridique assuré de voir reconnu dans le cadre social commun, certaines de ses demandes. Le rapport à soi structurellement inscrit dans la relation juridique est d'ordre universel dans la mesure où il doit, en principe, s'appliquer de la même manière à chaque sujet moralement responsable de la communauté. Enfin, la reconnaissance est aussi une forme de relation pratique à soi-même assurant la valeur sociale de son identité et ouvrant non plus seulement au respect de soi, mais aussi à l'estime sociale, ce qui «désigne l'attitude positive qu'un individu est capable d'adopter à l'égard de lui-même lorsqu'il est reconnu par les membres de sa communauté comme une personne d'un certain genre $^{7} »$. Pour Honneth, cette troisième voie dans la reconnaissance habilite l'individu à se rapporter positivement à ses qualités personnelles et singulières et à leurs capacités concrètes. C'est là une reprise de ce que Hegel avait entrepris de conceptualiser comme vie éthique ou éthicité. L'estime sociale est donc une forme de reconnaissance qui s'appuie sur des fins éthiques «culturelles » qui imprègnent les sociétés en ce qu'elles leur fournissent des valeurs de référence qui ne sont celles ni de l'amour ni du droit. La quête d'estime sociale se fonde sur la reconnaissance d'un état situé dans une nomenclature culturelle et "c'est la "valeur" de cet état - résultant de la contribution collective, socialement définie, qu'il apporte à la réalisation des fins de la société - qui détermine la valeur sociale de ses membres (...) Le passage à l'âge moderne ne signifie pas seulement la dissociation de la relation de reconnaissance juridique d'avec l'ordre hiérarchique de l'estime sociale; il soumet aussi celui-ci à un processus de transformation structurelle, porteur de luttes opiniâtres, parce que les innovations culturelles modifient aussi les conditions de validité des fins éthiques d'une sociétée "». 
4 La troisième sphère de reconnaissance nous invite donc à considérer l'autoréalisation sous l'angle de demandes d'accréditation édictées par des sujets depuis leurs singularités personnelles, mais attestées par des normes culturelles partagées (une estime sociale de soi pourrait-on dire ${ }^{9}$ ). Or, il nous semble également possible de considérer qu'il existe des demandes de reconnaissance qui, si elles s'approchent formellement d'une sollicitation visant l'estime sociale de soi, s'en distinguent néanmoins parce qu'elles se fondent sur la nécessité d'une attestation d'un sujet envisagé dans sa vie individuelle et/ou comme appartenant à une communauté restreinte. Peut-être pourrait-on avancer le syntagme « estime subjective de soi » afin de distinguer cette modalité de reconnaissance de l'estime sociale de soi dont les principes de validation ont un caractère plus universel à l'échelle de la société. Si la lutte pour la reconnaissance tend à être présentée par Honneth comme un parcours dans la mesure où, nous dit-il, un développement réussi de soi présuppose une certaine succession de formes de reconnaissance attachées aux trois sphères que nous venons de passer en revue (car c'est seulement ainsi que l'on serait en mesure de s'éprouver en tant qu'individu autonome, individualisé développant une pleine dignité), la reconnaissance complète d'une individualité en tant que personne totale ayant une identité propre passe vraisemblablement aussi par la reconnaissance intersubjective de certains attributs plus personnels.

\section{Reconnaissance et théorie des champs}

Dans l'immédiat, nous voudrions ouvrir une première discussion et envisager l'intérêt que nous pourrions avoir à saisir les différentes modalités de reconnaissance (confiance, respect et estime de soi) en lien avec une pluralité des formes de domination sociale et en relation avec des sphères sociales variées. Si la dignité, en tant qu'elle est «la confirmation par autrui de l'idée qu'un individu se fait de sa propre valeur ${ }^{10}$ ", s'édifie sans doute possible sur les trois sphères de la reconnaissance honnéthienne, il apparait tout aussi évident qu'il existe des espaces spécifiques qui peuvent être d'efficients pourvoyeurs d'interactions de reconnaissance. Les besoins et les affects sont à l'évidence largement pris en charge dans le cadre des relations familiales, amoureuses et amicales, la responsabilité morale étant assurée par des garants institutionnels. Quant aux capacités et aux qualités personnelles, on conçoit aisément qu'elles soient en lien avec des sphères d'activité hétérogènes et pas seulement liées au travail. La convocation de la théorie des champs pourrait, dans un premier temps, nous amener à prendre la pleine mesure des processus de différenciation du monde social et de ses enjeux, qui sont également des phénomènes de différenciation des identités sociales, des modes de connaissance du monde social et donc forcément aussi des modalités de reconnaissance qui leur sont liées, dans la mesure où « à chaque champ correspond un point de vue fondamental sur le monde qui crée sont objet propre et qui trouve en lui-même le principe de compréhension et d'explication convenant à cet objet ${ }^{11} »$. Elle peut ainsi nous permettre de mieux envisager la pluralité des modes de reconnaissance (sans en postuler une équivalente importance) et des formes de déni de dignité qui s'y greffent, notamment en ce qui concerne l'estime sociale de soi. Prenant l'exemple de la sphère professionnelle, Gérard Mauger insiste par exemple sur le fait qu' " en dehors du salaire, le travail en lui-même procure, en effet, des profits symboliques intrinsèques [dont de reconnaissance] (...). De 
même, l'effort fait pour s'approprier son travail, quel qu'il soit, ne peut manquer d'y attacher le travailleur qui, par son investissement, concourt à sa propre exploitation. Mais ces profits symboliques dépendent à la fois des conditions de travail [situations] et des dispositions des travailleurs ${ }^{12} »$.

6 Au regard de la reconnaissance, que nous apporterait la prise en compte de l'existence d'une pluralité des champs? Sans doute de montrer que les structures sociales ne se réduisent pas à l'État, à la sphère domestique ou même à celle du travail. Ces divers espaces se composent de champs et de sous-champs qui les différencient et offrent des conditions variées de mise en œuvre du lien social dont on imagine qu'elles spécifient aussi les formes de reconnaissance qui peuvent y être développées. Honneth convient qu'il est difficile de contrôler depuis la réalité phénoménale les pratiques positives de reconnaissance qui se donnent plus facilement à lire quand elles s'expriment négativement dans l'humiliation. En étant attentive aux statuts, aux positions, aux règles du jeu, aux intérêts spécifiques, aux formes de capitaux, la théorie des champs offre un appareillage conceptuel permettant de se saisir assez finement des rapports de domination et des phénomènes de (non-)reconnaissance qui lui sont attenants. Ces derniers peuvent d'ailleurs être dialectiquement liés aux premiers, dans la mesure où ceux qui occupent les positions dominantes dans le champ sont souvent les évaluateurs qui décernent la reconnaissance, mais leur propre reconnaissance peut aussi avoir partie liée aux sanctions négatives et aux dévaluations qu'ils peuvent attribuer en tant que ces opérations sont des formes d'affirmation de leur supériorité et de leur capital symbolique. Si la notion de capital symbolique a le réel avantage de permettre de désigner des formes sociales de distinction (reconnaissance) propres à des règles particulières qui sont celles d'un champ donné, elle autorise également à qualifier celles qui se greffent plus spécifiquement à des rapports de domination et peuvent prendre forme sur fond de mépris. Car de toutes les distributions, précise Pierre Bourdieu, "l'une des plus inégales et, sans doute, la plus cruelle est la répartition du capital symbolique, c'est-à-dire de l'importance sociale et des raisons de vivre ${ }^{13}$ ». La notion de capital symbolique permet ainsi de concevoir l'existence de hiérarchies dans les dignités et indignités et des modes de reconnaissance exercés "sous domination »; lesquels pour autant qu'ils soient des phénomènes de valorisation, n'en sont pas moins l'expression de rapports de force et de rapports de sens qui leur sont indexés.

7 Accepter d'être reconnu par telle instance ou tel sujet, c'est leur reconnaître a minima la légitimité de cette reconnaissance et, par là-même, naturaliser leur position et la hiérarchie dont elle dépend. En ce sens, l'acte de reconnaissance, comme l'éventuelle demande en amont, doivent être envisagés dans leur relation à l'ensemble des effets symboliques dont ils participent, et notamment ceux de la reproduction des positions dominantes dans tel ou tel champ ou, plus largement, au sein de l'espace social. Pour le dire autrement, le concept de capital symbolique invite à envisager les phénomènes de reconnaissance comme des boucles symboliques permettant, certes, d'accéder à des modalités variées de la dignité par le reconnu, mais aussi comme des opérations conjointes de légitimation du reconnaissant et de son éventuelle domination. Peu souligné dans les commentaires portant sur la théorie honnéthienne, le fait est que l'intersubjectivité de la reconnaissance est aussi une interdépendance potentiellement confirmatrice d'asymétries sociales: "Le capital symbolique assure des formes de domination qui impliquent la dépendance [relative] à l'égard de ceux qu'il permet de dominer : il [existe] dans et par l'estime, la reconnaissance, la croyance, le crédit, la 
confiance des autres, et il ne peut se perpétuer qu'aussi longtemps qu'il parvient à obtenir la croyance en son existence ${ }^{14} »$.

8 Mais au-delà même de pouvoir montrer l'existence de luttes spécifiques pour la reconnaissance, la théorie des champs autorise également à considérer ces luttes comme des rapports pratiques que les sujets entretiennent avec les règles du jeu du champ dans lequel elles s'inscrivent, et, de ce fait, contribuent par leurs luttes à maintenir la structure et l'existence du champ considéré. Se battre pour être reconnu dans certaines des formes de son "utilité sociale " contribue très certainement, en d'assez nombreuses occasions, à asseoir les formes de domination qui cadrent le principe même de cet investissement (une coïncidence réalisée de l'être et du devoir-être). Autrement dit, se faire reconnaitre sous certaines de ses caractéristiques ou compétences ne relève pas nécessairement d'une pratique exempte de toute ambiguïté au regard d'autres modes de subordination qui peuvent s'exercer dans le même mouvement, volontairement ou non. La théorie des champs permet ainsi d'envisager le répertoire des formes de reconnaissance et de mépris, de considérer les rapports dialectiques qu'elles entretiennent entre elles (renforcement, équilibrage, etc.) et de repérer leur importance respective dans l'image et l'estime de soi construites pas les sujets. Dans une perspective de mobilisation de la lutte pour la reconnaissance, ce dernier point semble important et pourrait expliquer, pour partie, pourquoi les dénis de reconnaissance ne déclenchent pas mécaniquement des combats pour recouvrer certaines formes de dignité. La pénibilité d'une humiliation peut être rendue supportable si, par ailleurs, il est des domaines de pratiques susceptibles d'apporter quelque modalité de reconnaissance et, par là, de relativiser l'amoindrissement du sujet en d'autres champs.

9 La théorie des champs permet donc d'injecter dans les processus de reconnaissance un rapport dialectique entre les structures sociales structurées (les champs) qui sont des histoires-faites-choses et des sujets porteurs d'identités singulières, qui peuvent être en quête de reconnaissance. Il faut enfin noter que si l'analyse en termes de champs contribue sans doute à considérer la reconnaissance comme un concept analysant " sans reste » la dignité, elle ne peut cependant remplir à elle seule cette mission. Pour le dire avec les mots de Bernard Lahire, la théorie des champs n'épuise pas la réalité de la différenciation sociale, la pluralité des mondes sociaux car « tout contexte pertinent d'activité [et de reconnaissance] n'est pas un champ ${ }^{15}$ ». Le hors-champ est bien évidemment couvert par les différentes sphères de la reconnaissance identifiées par Honneth et particulièrement celle de l'amour, mais il est aussi des univers sociaux faiblement structurés qui portent « des activités dans lesquelles nous nous inscrivons uniquement de manière temporaire (...) [et] qui ne sont pas assignables à des champs sociaux particuliers, parce que ces activités ne sont pas systématiquement organisées sous la forme d'espaces de positions et de luttes entre les différents agents occupant ces positions ${ }^{16} »$. En effet, il nous semble que certaines pratiques sociales qui relèvent en fait de domaines d'interaction, échappent à la fois au cadre de la théorie des champs, mais aussi à celle de la reconnaissance. Pourtant possiblement porteuses de formes reconnaissance, elles entrent néanmoins assez mal au sein de l'une ou l'autre des catégories de la modélisation tripartite proposée par Honneth. Si une partie de ces activités sont éventuellement indexables à la sphère de la solidarité ou envisageables comme relevant d'un champ particulier, modulo quelques contorsions analytiques, il en est d'autres qui restent orphelines de tout classement car, fondamentalement, l'espace 
social auquel elles se réfèrent est un espace très largement intériorisé, subjectivé, incorporé.

\section{Mépris et violence symbolique}

10 Nous venons de définir succinctement les phénomènes sociaux qu'Honneth désigne comme des faits de reconnaissance. Ces dynamiques de reconnaissance ont des pendants négatifs que le philosophe allemand définit comme des formes de mépris qui sont considérés par ailleurs comme autant de ferments des conflits sociaux. Les trois sphères normatives où se construisent les rapports pratiques des individus à euxmêmes conduisant à l'autoréalisation de soi, sont donc aussi des lieux possibles d'expériences déstabilisantes pour le sujet, directement liées au manque ou au déni de reconnaissance. Le mépris caractérise donc « un comportement qui est injuste en ce que, avant même d'atteindre les sujets dans leur liberté d'action ou de leur porter un préjudice matériel, il les blesse dans l'idée positive qu'ils ont pu acquérir d'eux-mêmes dans l'échange intersubjectif ${ }^{17}{ }^{7}$. C'est l'expérience morale d'un rabaissement et de l'impossibilité de se voir confirmer la valeur positive de soi, dans l'autre.

11 Le mépris est donc ce qui amène les acteurs sociaux à faire l'expérience concrète d'un déni de reconnaissance. Celui-ci peut prendre, dans la sphère de l'amour, la forme d'une atteinte à leur intégrité physique ou d'un déni dépréciatif de leurs sentiments et, par là, à leur capacité d'avoir une confiance élémentaire en eux-mêmes. Il peut également épouser la forme d'une relégation sociale par le refus de certains droits, mettant ainsi à mal le respect moral que les individus sont justement en droit de se porter. C'est le respect de soi qui se trouve alors affaibli en ce qu'il n'est plus possible de se considérer comme un partenaire d'interaction de même statut. Enfin, il existe un dernier mode de reconnaissance dont le mépris prive cette fois le sujet de sa valeur sociale. Ce sont, en ce dernier cas, des formes d'offense et d'humiliation qui refusent au sujet l'approbation de ce qu'il est en tant qu'il appartient à un groupe social, ou bien en tant qu'il est un individu singulier. L'individu ainsi blessé dans la relation à soi peut se sentir " personnellement visé » par ces formes d'abaissement culturelles qui lui font ressentir une certaine honte ou une certaine colère car "les modèles institutionnels de l'estime sociale se sont individualisés (...), leurs jugements de valeur portant [davantage] sur des traits individuels et non [plus seulement] collectifs ${ }^{18}$ ». Quand cette honte et cette colère sont interprétables comme des expériences partagées par un collectif, c'est alors, d'après Honneth, la possibilité d'une lutte sociale qui naît.

Ne peut-on pas, là aussi, convoquer certains apports conceptuels de la sociologie bourdieusienne? Le mépris semble en effet, à première vue, entretenir quelque accointance analytique avec le concept de violence symbolique. Que ce dernier serait-il en mesure de nous aider à penser s'agissant des phénomènes de déni de reconnaissance ? Reprenons avec Estelle Ferrarese les discussions que nous avons entamées supra: «En transformant autrui en instance, nous dit la politologue, c'est-à-dire en octroyant une légitimité à son acte de reconnaissance ou de déni de reconnaissance à venir, je lui reconnais le droit de m'infliger ou non un tort qui me placera dans l'impossibilité de nourrir un respect de moi-même. C'est en ce sens qu'un acte de reconnaissance implique toujours l'existence d'un pouvoir. Immanent à son champ d'apparition, celuici ne s'explique pas dans les termes d'un agir stratégique. Mais il naît du fait que celui à qui est adressée une demande de reconnaissance peut toujours refuser d'y répondre. 
Émettre une prétention à la reconnaissance implique d'accepter que l'autre use de cette possibilité19 ".

13 Cette assertion nous invite à creuser la question de l'illusio que Bourdieu définit comme "l'adhésion immédiate à la nécessité d'un champ ${ }^{20}$ ». Celle-ci peut être liée, comme le suggère Philippe Corcuff, à une quête du sens de soi et à l'impossibilité d'échapper aux "jeux de distinction entre les personnes et les groupes dans la quête d'une reconnaissance par les autres ». Citant les Méditations pascaliennes, il poursuit : « Nous somme au cœur de "la question de la légitimité d'une existence du droit d'un individu à se sentir justifié d'exister comme il existe". (...) “C'est la société, et elle seule, qui dispense, à des degrés différents, les justifications et les raisons d'exister" ${ }^{21} »$. On reconnaît là les postulats intersubjectifs et normatifs qui sont au fondement de la théorie de la reconnaissance, mais ce que suggère l'illusio, en accord avec la théorie des champs, c'est que cette nécessité pour le sujet d'être reconnu et auquel s'attache le risque du mépris, passe par un investissement dans des manières d'être occupé par le monde via une adhésion à des jeux sociaux auxquels nous avons intérêt à croire, dans la mesure où c'est à travers eux que nous pouvons obtenir gain de reconnaissance. Cela nous pousse à nous interroger sur le fait que l'autoréalisation (partielle) semble passer paradoxalement par une méconnaissance de la réalité sociale (notamment des règles des champs) et de sa vérité objective. Surtout, elle tend à tirer les phénomènes de reconnaissance vers des horizons relativement éloignés de ce que pourrait être une "vie bonne ", voyant en eux un travail " toujours recommencé, des individus et des groupes pour acquérir la reconnaissance des autres, dans la concurrence perpétuelle avec eux ; reconnaissance supposée, au final, largement vaine et illusoire ${ }^{22}$ ».

La violence symbolique reçoit, de ce fait, peu d'intérêt de la part des tenants de la théorie de la reconnaissance, puisqu'elle cherche surtout à attirer l'attention sur les aspects potentiellement négatifs de la reconnaissance alors qu'au sein de la théorie éponyme, elle est évidemment le pôle positif de l'action sociale. Ce qu'elle nous invite à effectuer c'est un recadrage de la reconnaissance et du mépris à l'aune d'un négativisme qui les conçoit possiblement comme participant d'une efficace visant l'acceptation des formes de domination susceptible de contribuer à la perpétuation de l'ordre établi. La violence symbolique, c'est l'incorporation par les sujets de schèmes perceptifs opérant une naturalisation des rapports sociaux, de sorte que leurs actions s'accordent avec leur connaissance, ou, pour être plus juste, avec leur méconnaissance des principes de la domination dont l'efficacité repose sur la reconnaissance que le dominé accorde au dominant. Autrement dit, le mépris qui s'exerce objectivement sur un sujet n'est pas forcément un acte le conduisant à faire une expérience morale de l'injustice et de l'offense, mais peut être aussi appréhendé par le sujet méprisé comme une interaction "normale ", en tant que celle-ci est normalisée par sa méconnaissance de l'acte effectif de domination qui s'exerce à son encontre. Cette pointe critique a, nous semble-t-il, été partiellement prise en considération par Honneth via la conceptualisation d'une idéologie de la reconnaissance (nous y reviendrons infra). Toutefois, vu au travers du prisme de la violence symbolique, l'aiguillon du mépris perd de son efficace mobilisatrice car il est atténué par l'intériorisation des processus de domination qui sont à l'origine du déni de reconnaissance. Ce que soulignent les notions d'illusio ou de violence symbolique, à leur manière, c'est le risque pour la théorie de la reconnaissance, même si elle accorde une forte importance aux phénomènes de mépris, de faire l'impasse sur des registres de l'exploitation, de la 
domination et des inégalités qui, s'ils entretiennent un rapport très étroit avec la reconnaissance et ses dénis, ont pour autant leur autonomie.

\section{Intersubjectivité et dispositionnalisme}

Chez le jeune Hegel, il est déjà entendu que la formation du «je » n'est effective entre sujets que «quand chacun des deux individus se trouve confirmé dans son activité propre par son vis-à-vis qu'il parvient corrélativement à se comprendre lui-même comme un "je" individualisé agissant de façon autonome ${ }^{23}$ ", car c'est là un des soubassements de sa théorie de la vie éthique. «Cette réflexion, précise Honneth, reste cependant tributaire des présupposés de la tradition métaphysique, dans la mesure où elle n'envisage pas la relation intersubjective comme un événement empirique survenant à l'intérieur du monde social, mais qu'elle la stylise sous la forme d'un mécanisme de formation mettant en jeu des intelligences singulières ${ }^{24} »$. La convocation de la sociologie de Mead sort la pensée hégélienne de son théoricisme et lui confère une orientation plus attentive à la réalité sociale. Ce dernier socle théorique est donc mobilisé par Honneth afin de reformuler la vision spéculative hégélienne de la lutte pour la reconnaissance depuis des bases empiriques, ouvrant la possibilité d'une analyse conceptuelle contrôlée sur des données de fait.

Si la convocation de la pragmatique meadienne peine toutefois à sortir du cadre de l'histoire de la pensée théorique et à convertir le travail d'Honneth en un exercice conceptuel «sur matériaux », elle permet néanmoins d'inscrire la discussion des écrits $d u$ « jeune Hegel » dans une perspective intersubjective plus réaliste et d'intégrer dans la compréhension du rapport d'interaction une dimension morale. Le recours à la pragmatique meadienne inscrit la perspective de la reconnaissance dans un cadre normatif qui convient pleinement au projet de philosophie sociale d'Honneth. Elle lui confère également une charge proprement sociale qui pourrait l'inviter à étendre son heuristique sociologique en deux directions distinctes, mais qui de facto ne sont pas mobilisées par le philosophe francfortois.

Le caractère intersubjectif (relationnel) des phénomènes de reconnaissance nous semble devoir être appréhendé plus avant dans son aspect incorporé (dispositionnel), c'est-à-dire lié à un passé-présent ayant trait à la socialisation et aux parcours biographiques des individus. Un complément profitable à l'approche intersubjective de la reconnaissance pourrait être en effet apporté par la mobilisation d'un dispositionnalisme critique attentif à la singularité et la pluralité des identités. La notion de disposition rend compte de l'existence de schèmes intériorisés, d'inclinations dynamiques et relationnelles qui sont des «économies psychiques » structurées sur des contraintes et des ressources collectives individualisées. Les dispositions sont fatalement plurielles, conditionnelles, i.e. modulées diachroniquement par les parcours biographiques et synchroniquement par les contextes. Fondamentalement, on a donc affaire à « des schèmes d'action, des manières de faire, de penser, de sentir et de dire adaptées (et parfois limitées) à des contextes sociaux spécifiques. [Les sujets] intériorisent des modes d'action, d'interaction, de réaction, d'appréciation, d'orientation, de perception, de catégorisation, etc., en entrant peu à peu dans des relations sociales d'interdépendance avec d'autres acteurs ou en entretenant, par la médiation, d'autres acteurs, des relations avec de multiples objets dont ils apprennent le ou les usages, le ou les modes d'appropriation ${ }^{25} »$. Le dispositionnalisme entretient 
ainsi quelque accointance avec le pragmatisme de Mead, lequel souligne la pluralité identitaire : «Les types de relation que nous entretenons varient suivant les différents individus ; nous sommes une chose pour un homme et une autre pour un autre. (...) Il existe ainsi une grande diversité de soi correspondant aux différentes réactions sociales. (...) Une personnalité multiple est en un sens normale ${ }^{26} \%$. Toutefois, en considérant que les sujets sont porteurs d'histoires-faites-corps (une identité sociale plurielle incarnée), cela nous autoriserait à reconsidérer les normes sociales de l'action de manière sensiblement différente que par le truchement du concept meadien d'autrui généralisé. Certes, celui-ci suppose un phénomène d'intériorisation des normes d'action par la socialisation, mais il tend également à considérer l'incorporation de ces règles culturelles uniquement comme une ressource d'ajustement positif à l'environnement, alors que celles-ci peuvent aussi être une manière de ressentir et d'agir qui peut fragiliser ou aller à l'encontre de la relation de reconnaissance. Si les normes intériorisées cadrent «à la fois quelles attentes [le sujet] peut légitimement adresser aux autres membres du groupe, et quelles obligations il est tenu de remplir à leur égard ${ }^{27} »$, elles ne sont pas (seulement) des actes réflexifs et d'alignement conformes aux attentes sociales. Elles sont également l'expression de logiques d'action qui peuvent, à un moment donné, être en décalage avec les exigences d'un environnement n'appelant plus de conformation à ces règles. On peut très bien imaginer qu'il y ait intériorisation des normes sociales d'action de l'autrui généralisé, mais que celles-ci, en certaines situations, face à des sujets singuliers (et pas la communauté toute entière), ne soient plus les logiques d'action primaires à mettre en œuvre. Acquérir l'identité d'un membre accepté de sa communauté ne veut pas forcément dire que la relation intersubjective de reconnaissance ait un caractère de systématicité. Considérer le sujet comme porteur de dispositions hétérogènes permettrait notamment d'envisager plus finement les situations de mépris. Être empêché de se rapporter positivement à soi peut venir d'un obstacle extérieur (e.g. ne pas être reconnu dans ce que l'on estime être ses droits), mais cet obstacle peut également être incorporé, se faire disposition ou penchant et affaiblir la capacité des individus à être reconnus.

Le postulat dispositionnaliste nous évite de supposer l'existence de pulsions intérieures ou d'un sens moral du soi transcendant. Honneth concède d'ailleurs que «l'attitude humaine de reconnaissance pourrait alors se comprendre comme un faisceau d'habitudes qui se sont rattachées, au cours du processus de socialisation [à une identité et] aux raisons révisables d'attribuer de la valeur à d'autres personnes ${ }^{28}$ ». Dans cette perspective, la reconnaissance est la conséquence d'une entente intersubjective entre des sujets porteurs de dispositions actualisées dans une situation donnée, le mépris étant le phénomène inverse : une mésentente intersubjective. Les conséquences de la reconnaissance ou du mépris peuvent être, nous dit-on, des accessions ou au contraire des atteintes plus ou moins durables à des identités plus ou moins dignes. Se sentir confiant, respecté, estimé et accéder à certaines formes stabilisées d'autoréalisation pourrait être alors conçu comme lié à une intériorisation qui ne soit pas juste ordonnée par le respect ou la dénégation de normes morales, mais relèverait de schèmes dispositionnels dessinant un ethos, c'est-à-dire "un ensemble objectivement systématique de dispositions à dimension éthique [et] de principes pratiques ${ }^{29}$ » qui lui sont corrélés. 
Quand on initie une interaction visant (ou non) une demande de reconnaissance on peut y rentrer avec des exigences normatives de dignité, mais on s'engage aussi depuis ce que l'on est dispositionnellement parlant, par exemple un sujet blessé car ayant été mésestimé à plusieurs reprises par le passé, ou au contraire un individu ayant une pleine confiance en soi. Honneth semble voir dans l'attente de reconnaissance le moteur essentiel de la lutte pour la réalisation de soi et de l'engagement dans les combats pour la dignité parce que son approche est normativement construite depuis cette exigence. Mais en réalité, l'aiguillon moral n'est qu'un des éléments de la dynamique de l'action (il en est le motif), mais ne peut être tenu pour la condition suffisante de la mise en œuvre de cette action. Précisant que la conscience n'est pas un préalable de l'acte social, mais que ce dernier en est la condition préalable (principe de l'intersubjectivité qui, moralisé, induit que la reconnaissance précède la connaissance), Honneth semble néanmoins parfois renverser, le principe au nom de la normativité du progrès moral. S'engager nécessite pourtant des capacités d'action qui ne procèdent pas directement de l'exigence morale, mais qui sont en lien avec des identités sociales éprouvées, dynamiques et qui confèrent ou non des capabilités aux sujets (notamment celle de se raconter). Une sociologie dispositionnaliste considère potentiellement que ces «blocages intérieurs" et "inhibitions psychiques" sont des «plis» de la personnalité qui sont en relation avec des expériences de socialisation passées et peuvent amoindrir l'aptitude des sujets à se sentir reconnu. Elle permet ainsi d'articuler des conditions sociales d'existence objectives avec des formes de subjectivation qui ne se résument pas à un idéalisme normatif, c'est-à-dire à une construction morale de la réalité (il n'y aurait que des points de vue éthique sur le monde), mais d'ancrer identitairement les demandes de reconnaissance. Ce qu'un dispositionnalisme critique nous apprend et nous permet de ne pas perdre de vue, c'est que la dignité nécessite des conditions de réalisation de soi qui peuvent être fragiles, voir inexistantes, car elles tiennent fondamentalement à des échanges intersubjectifs eux-mêmes cadrés par des rapports sociaux qui en définissent la portée. La reconnaissance, fût-elle considérée comme une souche morale "universelle », ne se soustrait pas pour autant à l'ordre social ordinaire et aux orientations qu'il impose, notamment en pesant sur les conditions matérielles d'existence. Le sujet est porteur de schèmes de perception et d'appréciation, de principes de vision et de division qui lui donnent les moyens d'opérer des actes pratiques de connaissance, mais aussi de reconnaissance et de mépris. Il s'agit alors de ne pas oublier que ceux-ci ne relèvent pas toujours d'un calcul rationnel ou moral, mais sont aussi, pour partie, cadrés par des contraintes structurales dont ils sont les produits et qui conditionnent les conduites. Autrement dit, la reconnaissance et son déni sont des agir qui peuvent également relever d'un sens pratique permettant au sujet de se comporter comme il faut au regard de situations dont les règles de conduite peuvent être très éloignées de l'exigence de la reconnaissance, ou bien même, réclamer certains actes de mépris.

\section{Singularités plurielles et estime subjective de soi}

21 Le principe de la pluralité dispositionnelle ouvre également d'autres perspectives quant à l'opportunité de saisir les demandes de reconnaissance plus singulières que nous avons évoquées supra. Commentant les spéculations de Mead, Honneth souligne que pour que le sujet puisse prendre envers lui-même une attitude positive à partir de 
qualités qui le distinguent de ses partenaires d'interaction, il faudrait « une forme de reconnaissance mutuelle qui confirme chaque individu non seulement comme membre de sa communauté, mais aussi comme un sujet porteur d'une histoire individuelle ». Il précise que Mead rejoint sur ce point également Hegel « lorsqu'il établit que la relation de reconnaissance juridique est encore incomplète, dans la mesure où elle ne peut donner une expression positive des différences individuelles entre les citoyens d'une communauté30 ». En termes meadiens, il s'agit donc de prendre en compte le « je » qui entraîne des réactions de l'individu face à la communauté telle qu'elle apparaît au travers de son expérience et dont Honneth affirme qu'elle est une force inconsciente opposée au «moi », mais aussi une demande de reconnaissance spécifique. Dans cette perspective, l'autoréalisation passe par la reconnaissance d'une personnalité unique et singulière, une confirmation éthique de soi par soi, mais en accord avec les valeurs d'une collectivité (ou d'un individu) qui permet de s'assurer « de la signification sociale de ses capacités individuelles ${ }^{31}$ ».

Comme nous l'avons déjà vu, cela débouche sur l'existence de la troisième relation éthique de reconnaissance mutuelle identifiée plus haut comme estime sociale de soi. Honneth propose de qualifier de solidarité la relation d'interaction par laquelle les sujets «s'intéressent à l'itinéraire personnel de leur vis-à-vis parce qu'ils ont établis entre eux des liens d'estime symétrique ${ }^{32} »$. Mais au fil de son argumentation, il va encore plus loin et souligne que l'horizon des valeurs culturelles des sociétés capitalistes avancées tend sensiblement à se pluraliser et donc, par conséquent, à individualiser la reconnaissance fondée sur l'estime sociale. Il en vient alors à affirmer que «ces idées directrices, devenues abstraites, ne fournissent pas un système de références universellement valide, à partir duquel on pourrait mesurer directement la valeur sociale des qualités et des capacités particulières : elles demandent au contraire à être concrétisées par des interprétations culturelles complémentaires, avant de pouvoir être appliquées dans cette sphère de la reconnaissance. C'est pourquoi la valeur attribuée aux différentes formes d'autoréalisation, mais aussi la définition même des qualités et des capacités correspondantes, dépendent fondamentalement des interprétations qui gouvernent la perception des fins sociales à un moment particulier de l'histoire ${ }^{33} »$. Honneth remarque ainsi que les rapports d'estime sociale s'inscrivent potentiellement dans des luttes pour la reconnaissance portées par des groupes sociaux qui tentent de valoriser symboliquement leurs spécificités identitaires et pratiques en tant qu'elles sont susceptibles de jouer un rôle positif non négligeable pour la société. Mais sans doute est-il également profitable de mettre en avant le fait que la confirmation des sujets dans leurs particularités individuelles prend également appui sur des phénomènes qui ne valorisent pas des spécificités collectives, mais bien individuelles, c'est-à-dire permettant au sujet de s'identifier dans sa subjectivité propre, sans que celle-ci ne soit indexée à des qualités partagées. Ce sont, dans ce cas, des contributions individuelles qui sont portées au jugement d'autrui et dont les fins sociales revendiquées n'ont pas toujours vocation à être des considérations sociales universelles.

Comme nous l'avons déjà souligné, l'estime sociale de soi se fait alors estime subjective de soi en tant que les individus sont potentiellement en mesure d'éprouver le sentiment de leur propre valeur et, pour emprunter les mots de Bourdieu de se sentir justifiés d'exister comme ils existent. Cette dialectique du "même » et de la différence est d'ailleurs, d'après Ferrarese, l'un des axes problématiques essentiel de toutes les 
théories de la reconnaissance: «S'agit-il de reconnaitre l'autre en tant qu'il est un autre moi-même, mon égal, ou bien en tant qu'il est autre, c'est-à-dire dans sa différence ? $^{34}$ ». Même et différent, c'est-à-dire un sujet à la fois singulier car pluriel, qui s'est construit depuis des appartenances et des rôles correspondant aux différents espaces de socialisation qu'il a pu arpenter et qui peuvent être animés par des ordres de reconnaissance variés. La reconnaissance a donc lieu quand un individu reçoit de tiers le moyen de se rapporter positivement à sa personne, en tant qu'individu désiré/ désirant, membre d'une société forgée en droit, élément solidaire d'une communauté et individu singulier. Dans cette perspective, la relation de reconnaissance peut aussi être une interaction confirmant un individu singulier/pluriel en ses attributs les plus personnels qui, s'ils sont évidemment collectifs par construction, sont en même temps individués du fait de leur incorporation et, de ce fait, peuvent ne se réclamer d'une quelconque utilité sociale. La singularité plurielle des sujets dans leur composante identitaire nous invite à considérer que si la constitution de l'identité personnelle est indexée à la reconnaissance de la dignité, elle ne s'y résume pas car, sous sa forme concrète, le rapport positif à soi est toujours un rapport à sa propre identité personnelle, intersubjectivement constituée et travaillée par la socialisation.

À l'instar de Mead qui précise qu'«il y a des parties de soi qui n'existent que par rapport à lui-même" mais qui par ailleurs peuvent s'harmoniser dans un "soi complet ${ }^{35}$ ", Emmanuel Renault évoque également l'unification des différentes strates de l'identité biographique car notre identité peut se concevoir comme l'ensemble relativement intégré des représentations de soi et des caractéristiques sociales/ psychologiques dont on se sent porteur et qui font également notre valeur en tant qu'individu. Si la reconnaissance porte sur la dignité, elle touche également l'identité et sa variété, tout comme son négatif : déni de dignité ou mépris de l'identité. Autrement dit, le rapport positif à soi tient à la confirmation positive de son identité personnelle et suppose ainsi des formes de reconnaissance qui ne peuvent seulement se résumer aux trois sphères de la reconnaissance honnéthienne. L'objet concret du besoin de reconnaissance "n'exprime pas seulement l'exigence d'un comportement juste à mon égard, c'est-à-dire d'un traitement qui prenne la mesure de ma dignité, de ma valeur universelle en tant que personne humaine sensible, responsable et sociable, il exprime également le besoin d'être reconnu comme cet individu particulier que je suis dans la vie ordinaire sous le masque des différentes identités que je porte dans l'interaction sociale ». Et d'ajouter : «Pour que le rapport positif à soi qui caractérise l'identité ne soit pas remis en cause par la pluralité des identités, il faut que ces différentes identités soient homogénéisables, que les différentes normes qu'elles comportent soient compatibles ${ }^{36} »$. En d'autres termes, le rapport positif à soi se trouve indexé à une double exigence : d'une part, être en mesure de faire reconnaître la dimension plurielle de son identité, et, d'autre part, maintenir une certaine homogénéité de sa singularité identitaire et faire en sorte que les différentes facettes de soi entretiennent un rapport non conflictuel du point de vue des valeurs qu'elles impliquent.

Une approche dispositionnaliste peut, nous semble-t-il, rendre finement compte de ces phénomènes de reconnaissance identitaire. Elle intègre en effet la possibilité de processus variés de socialisation et d'individuation du social incorporé dans des dispositions différenciées, mais conçoit aussi que l'intégration de ces dispositions ne soit pas uniquement un phénomène de transaction interne, mais de nature intersubjective. Les dispositions en tant que traits identitaires n'ont pas de caractère nécessairement permanent et elles entrent dans la composition du soi également en fonction des 
espaces et sujets sociaux fréquentés (couplage, activation, mis en veille, etc.). L'unification des différentes facettes de soi n'est donc pas tant un arrangement strictement individuel, qu'un phénomène de mise en compatibilité de sa singularité plurielle avec ses environnements et partenaires sociaux. Dans le langage de l'éthique de la reconnaissance, l'intériorisation des effets positifs de la reconnaissance de telle ou telle propriété personnelle conduit à l'estime subjective de soi et tend, dans le même mouvement, à intégrer ces propriétés à un soi unifié. On peut alors penser que l'itération de la confirmation de la valeur personnelle via la reconnaissance de traits identitaires singuliers peut engendrer des dispositions « qui constituent de nouveaux motifs de développement de ces capacités et cela les intègre toujours plus étroitement à la représentation du $\operatorname{soi}^{37}$ ». Évidemment, ce phénomène de mise en dispositions et de renforcement identitaire peut également s'appuyer sur le versant négatif du mépris. Dans ce cas, le sujet qui voit son investissement émotionnel sans cesse contrecarré peut se trouver globalement fragilisé dans l'unité de soi, et pas seulement "touché » en certains endroits de sa personne. La récurrence d'un déni de reconnaissance peut ainsi se cristalliser en des ambitions de reconnaissance et des désirs d'accomplissement sérieusement révisées à la baisse afin de s'ajuster à leur chance de succès. Le degré de satisfaction intime que connaissent les différents sujets dépend ainsi largement du degré auquel les espaces sociaux qu'ils arpentent favorisent ou non l'épanouissement de leurs dispositions et notamment de celles à être reconnu. En ce domaine de la reconnaissance subjective de soi, le pouvoir social tient à la possibilité d'imposer comme plus légitimes que d'autres, des ordres de reconnaissance propices à la valorisation de caractéristiques identitaires de certains sujets et de leur faciliter ainsi l'accumulation d'un capital symbolique de reconnaissance.

\section{Idéologie de la reconnaissance et contradictions paradoxales}

Honneth évoque également l'existence d'idéologies de la reconnaissance dont la logique est de mobiliser la reconnaissance à des fins stratégiques, invitant les individus à se livrer à des activités inédites pour eux, vers lesquelles ils ne seraient pas allés sans la promesse de reconnaissance qui les accompagne. Or cette promesse n'est tenue qu'en apparence. La culture affirmative de la reconnaissance ne travaille ni à l'autonomie des individus, ni à l'élargissement du pouvoir des sujets sociaux, mais, symboliquement accompagnée des représentations idéologiques de la reconnaissance, elle concourt à la mise en conformité des individus aux nouvelles exigences du système : "Les individus sont poussés à adopter, au travers de processus de reconnaissance mutuelle, un rapport à soi spécifique qui les incite à assumer de leur plein gré des tâches et des devoirs servant la société. (...) Reconnaître quelqu'un signifie alors l'amener, par des sommations répétées et assénées de manière rituelle, à adopter exactement le rapport à soi qui convient au système établi d'attentes de comportement ${ }^{38}$ ".

Dans La lutte pour la reconnaissance, la reconnaissance était exclusivement considérée comme l'envers des pratiques d'assujettissement. Or ce que conceptualise Honneth dans La société du mépris, c'est le rôle potentiel qu'elle peut jouer dans la domination sociale par la constitution et le maintien "d'un rapport à soi individuel parfaitement inséré dans le système de la division du travail dominante ${ }^{39}$ ». Les idéologies de la reconnaissance visent donc à conformer comportements et attitudes à des exigences 
fonctionnelles. La reconnaissance sociale est donc potentiellement pourvoyeuse d'effets idéologiques visant à mettre en conformité les individus avec le système. Pour ce faire, les idéologies de la reconnaissance travaillent à l'inclusion des sujets qu'elles souhaitent enrôler (et non à leur exclusion comme dans le cas du mépris). Elles se rendent crédibles à leurs yeux et établissent des énoncés de valeur réalistes et différenciés « au sens où ils doivent renvoyer de manière signifiante à des aptitudes ou à des vertus réelles chez leurs destinataires ${ }^{40} »$. Partant de ces impératifs, elles promettent, en référence à ces qualités ou via des imputations d'identité positive ad hoc, de reconnaître les sujets qui ont été ainsi motivés rationnellement et comptent bénéficier, du fait de leur engagement, d'une estime rehaussée de leur personne, alors que l'objectif est avant tout de leur imposer des tâches qu'ils n'auraient autrement assumées. Outre ces trois conditions quant aux conditions indispensables sous lesquelles les idéologies de la reconnaissance sont potentiellement efficaces, Honneth précise que l'efficience passe également par la nécessité que «quelque chose dans le monde physique des faits institutionnels ou des manières de se comporter doit changer pour que le destinataire puisse être effectivement convaincu d'être reconnu d'une nouvelle manière ${ }^{41} »$.

Le philosophe allemand décrit les dynamiques idéologiques de la reconnaissance comme relevant de contradictions paradoxales en tant qu'elles sont porteuses de progrès normatifs élargissant les possibilités d'épanouissement personnel et de promesses d'autoréalisation, mais aussi, dans le même mouvement, en tant qu'elles détournent ces opportunités à des fins instrumentales et instituent de nouvelles contraintes freinant ces formes d'autoréalisation. Leur fin est d'enrôler d'une autre manière les sujets dans le circuit de la production, de la consommation et de la reproduction du système capitaliste. La quête de reconnaissance est alors intégrée "dans le "profil" institutionnalisé sur lequel se fonde la reproduction sociale [et ce, au point que ces aspirations normatives] ont perdu leur finalité interne et sont devenues un principe de légitimation du système ${ }^{42}$ ". L'idéologie de la reconnaissance est aussi assurée d'une pleine efficacité parce qu'elle correspond à la forme la plus saillante de l'individualisme contemporain, typique des sociétés hautement différenciées et largement orienté vers l'idéal de la réalisation de soi.

La diversification des parcours biographiques, l'augmentation du niveau de certification scolaire, la culture de la consommation et du loisir, l'expansion de l'idéal romantique de vie, etc., sont autant de dynamiques qui, se couplant, ont donné naissance à une forme d'individualisme guidée par un principe d'épanouissement personnel. Dans la mesure où une partie de plus en plus importante de la population tend à « interpréter sa propre biographie non plus comme un processus figé et linéaire d'adoption séquentielle de rôle familiaux et professionnels, mais comme une opportunité de réaliser sa propre personnalité de manière expérimentale ${ }^{43}$ ", l'individu contemporain est sommé de se comporter en entrepreneur de soi-même. Il doit veiller à la production de son propre capital humain, optimiser ses investissements relationnels, cognitifs, affectifs, se créer des opportunités de formation, d'emploi, de satisfaction de ses désirs et de valorisation de soi, etc. En cela, il est parfaitement adapté aux nouvelles exigences du capitalisme qui organise son activité sur la base de projets et qui réussissent à mobiliser des ressources motivationnelles inédites. S'appuyant notamment sur l'amoindrissement des identités et des solidarités de classe, 
au bénéfice des identités individuelles, l'idéologie de la reconnaissance survalorise l'initiative, la mobilité, la réussite personnelle et l'autoréalisation.

Il faut noter qu'avec le thème de l'idéologie de la reconnaissance, Honneth prend davantage en compte la question de la méconnaissance et du " faux adressage ». Il précise ainsi qu'il existe "une reconnaissance s'opérant de manière déplacée, trompeuse et génératrice de loyauté ${ }^{44} »$. Les formes idéologiques de la reconnaissance se fondent, ajoute-t-il, sur « la répétition [c'est nous qui soulignons] constante des mêmes formules de la reconnaissance [et par là même parviennent] à créer sans recourir à la contrainte un sentiment d'estime de soi incitant à des formes de soumission volontaire ». Aussi, pour être pleinement efficaces, les idéologies de la reconnaissance doivent s'appuyer sur « des motifs évaluatifs rattachés de manière interne à notre horizon de valeurs » et ainsi veiller «à assurer une disposition motivationnelle [c'est nous qui soulignons] afin que s'effectuent sans résistance les devoirs et les tâches attendus ${ }^{45}$ ». Phénomènes de méconnaissance, ancrages et stabilisations de dispositions, mises en correspondance avec des schèmes de pensée : on reconnaît là quelques-unes des thématiques d'une sociologie pour laquelle l'efficace de la lutte symbolique est liée au phénomène de nécessités externes (e.g. de discours) rencontrant des nécessités internes de type dispositionnel. L'idéologie perd ainsi son caractère fonctionnaliste et non contradictoire pour affirmer une nature éminemment plus sociale et historique, c'està-dire à la fois inscrite dans les corps et dans les choses. Si les effets de l'idéologie sont généralement perçus comme étant opaques, c'est moins parce qu'ils se fondent sur une nécessité mensongère que parce qu'ils sont, tout comme l'action sociale, le produit d'une rencontre entre des dispositions pénétrées par l'histoire dont elles sont le produit et des entités symboliques elles aussi marquées par cette histoire et que cette confrontation s'accomplit au surplus sous la contrainte des structures qui ont produit cette histoire. Son efficience ne relève donc pas de la puissance persuasive des forces propagandistes ou de leur capacité à poser le voile de la fausse conscience sur les esprits malléables des individus, mais tient au "synchronisme " entre des schèmes cognitifs de perception incorporés et des structures objectives. Ce sont ces formes d'accointance intégratrice qui rendent alors possibles l'adhésion des individus à l'idéologie et assurent leur intégration morale et pratique. Le fait qu'une idéologie soit crédible et convaincante ne tient pas à un acte de conscience en réception qui validerait le message porté, mais au fait qu'elle puisse «adhérer » à des affects positifs (e.g. le sentiment positif de soi), relayer des croyances entendues comme dispositions à croire et s'actualiser dans des dispositions à agir. Les idéologies de la reconnaissance sont alors "acceptées » tacitement comme cadres d'interprétation possibles d'un rapport au monde qui se trouverait en conformité avec les attendus des individus qui produisent ces cadres. Elles sont donc fondamentalement l'expression d'une violence symbolique dont le principe de rendement repose sur une adhésion extorquée aux dominés via l'imposition douce d'un arbitraire qui légitime l'ordre social qui en fait justement des dominés. Les idéologies constituent donc elles-mêmes l'instrument et l'expression des «luttes pour la reconnaissance et pour le pouvoir symbolique, c'est-àdire pour l'imposition des principes de division, de connaissance et de reconnaissance ${ }^{46} »$.

31 Le dispositionnalisme critique rencontre ici les attendus d'une philosophie sociale qui, selon la perspective d'Honneth "est tributaire, conformément à son intérêt de connaissance, de critères généraux sur ce qu'est la normalité de la vie sociale, dont la validité ne peut plus être garantie seulement indirectement par des postulats 
anthropologiques, son existence à venir dépend désormais entièrement de la possibilité de fonder une éthique formelle ${ }^{47}$ ». C'est bien de la possibilité de pouvoir fonder des jugements éthiques depuis un principe supérieur commun concernant la vie bonne et mobilisés dans la pratique (i.e. à partir d'une «normalité empiriquement opérante ») que dépendent à la fois la philosophie et la lutte sociales, ainsi que leur orientation vers l'émancipation. Honneth critique ainsi, aussi bien l'esthétique philosophique de Theodor Adorno que le freudo-marxisme d'Herbert Marcuse car les exigences normatives dont ils se prévalent sont «dissociées de toute analyse des conflits de la société actuelle et de leurs implications morales ${ }^{48}$ ». Le dispositionnalisme critique nous enjoint à considérer que la lutte pour la reconnaissance, à un niveau individuel, est liée à l'existence d'un ethos singulier, entendu comme une disposition éthique à la reconnaissance dont l'incorporation est évidemment liée à des formes de socialisation primaires (relevant notamment, dans l'enfance, de la sphère de l'amour), mais aussi secondaires. Pour prétendre à la reconnaissance, encore faut-il que le sujet ait l'«aptitude " de cette "conscience morale ». Au sein de ses travaux séminaux sur la reconnaissance, Honneth distingue souvent les analyses critiques qui s'appuient, parfois implicitement, sur l'idée normative d'un ordre social juste, de celles (dont la sienne) qui mettent en avant le principe de réalisation de soi et des conditions de possibilités d'une formation positive de l'identité. Il convient toutefois, avec Karl Marx, que cette opposition qui vaut peut-être en théorie perd de sa valeur heuristique sur le plan empirique: "L'apport particulier de Marx était peut-être, à ses propres yeux, d'avoir pu démontrer que l'injustice sociale représente simultanément une pathologie sociale. C'est-à-dire qu'il a identifié le fait même de l'injustice, liée à l'exploitation, à une pathologie sociale, qui s'exprime à travers l'aliénation, soit le fait que nous sommes tous, et pas seulement le prolétariat, rendus étrangers à nos propres conditions de vie, à la nature et à nous-mêmes ${ }^{49}$ ». Certaines de ses réflexions plus récentes tendent en effet à reconnaître que les principes de justice au travers desquels les dominés jugent moralement l'ordre social sont contenus " dans des sentiments d'injustice typiques, plus que dans des principes axiologiques formulables positivement ${ }^{50}$ ». Mobiliser cette disposition éthique à la reconnaissance ne va donc pas de soi et le philosophe allemand concède que «les modes de représentation des sentiments d'injustice sociale, ne sont pas, comme on le suppose trop souvent, à la libre disposition des sujets concernés, mais (...) sont influencés et déterminés par de multiples mécanismes de domination de classe. Ces processus de contrôle de la conscience morale ont pour tâche de réprimer assez tôt l'expression des sentiments d'injustice, pour que le consensus de la domination sociale ne se retrouve pas remis en cause. Aussi faut-il voir dans ces techniques de contrôle des stratégies qui assurent l'hégémonie culturelle de la classe dominante en limitant implicitement les possibilités de formuler les expériences d'injustice ${ }^{51} »$.

Il nous semble qu'un dispositionnalisme critique nous invite justement à ne pas opposer justice sociale et reconnaissance qui, si elles ne répondent pas aux mêmes sources normatives, ne sont pas pour autant incompatibles. Elles ne sont certes pas équivalentes, mais peuvent être considérées comme largement complémentaires, en ce sens qu'elles décrivent des phénomènes sociaux identiques à des niveaux analyticopratiques différents. La démarche dispositionnaliste permet de coupler ces approches et de saisir dans un même mouvement les phénomènes d'injustice, de mépris (et de pathologie sociale) en ce qu'ils participent pour le sujet d'une même réalité concrète. Ce sont bien les rapports sociaux et les formes de socialisation qui, fussent-ils 
différenciés, cadrent de facto les manières d'être, de faire et de ressentir des individus. Les conditions sociales d'existence, préparent ainsi les demandes de reconnaissance et les rapports que les personnes entretiennent à cette nécessité justificative de soi. Les inégalités sociales engendrent inévitablement des inégalités sous l'angle de la reconnaissance et cette « répartition inégale de la dignité sociale limite drastiquement la possibilité de développer un sentiment de respect de soi ${ }^{52} »$.

Réclamer l'égalité quant aux conditions de vie est une forme de demande de justice qui touche aussi aux conditions de possibilité de réalisation de soi; revendiquer une reconnaissance pleine et entière et maîtriser son devenir sur la base de la cohérence d'un moi unifié, c'est en même temps requérir les conditions d'une "vie bonne ». Pour le dire différemment, l'éthique de la reconnaissance est une des manières possibles de mettre en œuvre une morale pratique dont les exigences rencontrent nécessairement celles de la justice sociale et du combat contre les formes de domination qui sont celles de l'ordre social capitaliste. Maintenir ce rapport dialectique, aussi bien en théorie qu'en pratique permet de répondre normativement et localement aux formes de domination fonctionnelle des rapports sociaux, notamment de production, qui ont l'habitude de faire taire les demandes de réparation de l'injustice sociale par des « compensations conformes à l'esprit du capitalisme, c'est-à-dire par l'attribution individuelles de ressources de temps et d'argent $t^{53}$ \%. Les phénomènes d'individualisation ont réussi en bien des espaces sociaux à briser les collectifs, leurs solidarités et leurs potentiels mobilisateurs susceptibles de répondre aux injustices dont sont victimes leurs membres. Face à ce constat de sérialisation des intérêts, le fait de se prévaloir des principes normatifs de la reconnaissance peut contribuer à réarmer la critique à un niveau individuel (celui de la classe probable) et à donner quelques raisons pratiques à réagir à ceux dont on amoindrit la vie. Cela permet «dans la dimension privée d'une action prépolitique, voire dans la sphère isolée de la pensée personnelle, [de] réévaluer symboliquement sa propre activité (...) [et de développer] une contre-culture du respect compensatoire ${ }^{54}$ " permettant de préserver une dignité minimale et éventuellement de porter le fer en son nom.

Mais, par ailleurs, quand le combat devient collectif (i.e. qu'il est celui d'une classe mobilisée) et quand le capitalisme prévoit de s'amender par des dédommagements fondés sur des rétributions symboliques faussement reconnaissantes, faire valoir les aspects redistributifs à plus grande échelle reste une forme essentielle de la conflictualité sociale. Les logiques de subordination ne sont pas économiques ou identitaires, mais se renforcent mutuellement. La réalisation de soi, des identités et des différences paraît des plus difficiles sans une justice sociale susceptible d'établir les conditions de possibilité d'une «vie bonne » et donc de conditions sociales d'existence ad hoc: " prendre en considération les effets des rapports structurels de domination sur l'existence individuelle ${ }^{55}$ » se présente alors comme une évidence. La dignité en général, et l'estime social de soi en particulier, ne sauraient prendre véritablement corps sous un régime socialement inégalitaire qui exclue, désaffilie, précarise et disqualifie. Les deux registres sont d'ailleurs d'autant plus essentiels qu'ils permettent de conserver une efficacité conflictualiste dans ces moments de plus en plus fréquents où, sous l'effet des contradictions paradoxales évoquées supra, les aspects négatifs peuvent se mêler aux aspects positifs, la subordination à l'autonomie, les dommages aux améliorations, etc. Pouvoir s'appuyer sur l'une ou l'autre de ces positions revendicatives permet de conserver une acuité critique qui ne se laisse pas désarmer 
par les fausses rétributions d'un capitalisme rendu «éthique » par l'enrôlement de principes allant formellement dans le sens d'une autonomisation du sujet.

La théorie de la reconnaissance constitue une base conceptuelle intéressante pour envisager une analyse critique du lien social contemporain. Sa matrice intersubjective apporte un caractère sociologique à son heuristique, en même temps qu'une normativité anthropologique correspondant à des privations et des afflictions effectives et qui pose les fondements d'une saisie critique du monde tendant vers l'émancipation (i.e. restituant le sujet à lui-même). La normativité de la théorie de la reconnaissance semble donc emprunter, en première lecture, à la fois à une critique herméneutique émanant du terrain, ainsi qu'à une critique rationnelle établissant des normes universelles transcendant les contextes. Ce point d'appui normatif est considéré comme permettant de faire dialoguer sciences et philosophie sociales, dont on sait que le programme de recherche interdisciplinaire de l'École de Francfort n'a réussi que partiellement à les rapprocher. Cela autoriserait ainsi à rassembler en une démarche critique, prises morales et potentialités analytiques empiriquement fondées. Toutefois, l'articulation de ce principe normatif à la réalité des faits de reconnaissance n'est pas un rapport d'évidence. Ce que permet notamment le dispositionnalisme depuis lequel nous avons discuté la théorie de la reconnaissance, c'est de recadrer reconnaissance et mépris à l'aune d'un négativisme qui les conçoit possiblement comme participant d'une efficace visant l'acceptation des formes de domination susceptibles de contribuer à la perpétuation de l'ordre établi. La violence symbolique, c'est l'incorporation par les sujets de schèmes perceptifs opérant une naturalisation des rapports sociaux, de sorte que leurs actions s'accordent avec leur connaissance, ou, pour être plus juste, avec leur méconnaissance des principes de la domination dont l'efficacité repose sur la reconnaissance que le dominé accorde au dominant. Pour Olivier Voirol, cette vision désenchantée des luttes symboliques se rapproche trop de la dynamique de l'intérêt et il lui reproche sa logique de l'action et du conflit « qui repose sur la maximisation de l'utilité et cela même si elle n'est pas pleinement consciente et transparente à l'acteur ${ }^{56}$ ». Le dissensus vient ici du caractère normatif de la théorie de la reconnaissance qui intègre à la théorie un principe d'émancipation, alors qu'au sein de la sociologie de la domination, cette dimension normative est largement implicite à la critique de l'ordre social. Et porter le fer contre la sociologie bourdieusienne en considérant qu'elle ne rend compte des processus de constitution et de reproduction de la valeur symbolique que depuis une réduction de type utilitariste, c'est juste oublier que la reconnaissance est socialement construite : «Alors que la méconnaissance de la nécessité enferme une forme de reconnaissance de la nécessité, et sans doute la plus absolue, la plus totale, puisqu'elle s'ignore comme telle, la connaissance de la nécessité n'implique pas du tout la nécessité de cette reconnaissance ${ }^{57}$ ». 


\section{NOTES}

1. Paul Ricœur, Parcours de la reconnaissance. Trois études, Paris, Gallimard, 2004 ;

Emmanuel Renault, L'expérience de l'injustice. Reconnaissance et clinique de l'injustice, Paris, La Découverte, 2004 ; Nancy Fraser, Qu'est-ce que la justice sociale? Reconnaissance et redistribution, Paris, La Découverte, 2005 ; Eva Illouz, Les sentiments du capitalisme, Paris, Seuil, 2006 ; Alain Caillé (dir.), La quête de la reconnaissance. Nouveau phénomène social total, Paris, La Découverte, 2007 ; Jean-Paul Payet et Alain Battegay (dir.), La reconnaissance à l'épreuve. Explorations socio-anthropologiques, Villeneuve d'Ascq, Presses universitaires du Septentrion, 2008 ; Alain Caillé et Christian Lazzeri (dir.), La Reconnaissance aujourd'hui, Paris, CNRS éditions, 2009 ; Christian Lazzeri et Soraya Nour (dir.), Reconnaissance, identité et intégration sociale, Paris, Presses universitaires de Paris Ouest, 2009, etc

2. Axel Honneth, La lutte pour la reconnaissance, Paris, Cerf, 2000 ; Axel Honneth, La société du mépris. Vers une nouvelle théorie critique, Paris, La Découverte, 2006 ; Axel Honneth, La réification. Petit traité de Théorie critique, Paris, Gallimard, 2007 ; Axel Honneth, Les pathologies de la liberté. Une réactualisation de la philosophie du droit de Hegel, Paris, La Découverte, 2008.

3. A. Honneth, 2000, op. cit., p. 11.

4. Ibid., p. 207.

5. A. Caillé, op. cit. ; A Caillé et C. Lazzeri, op. cit.

6. A. Honneth, 2000, op. cit., p. 117.

7. Ibid., p. 97.

8. Ibid., p. 150-151.

9. E. Renault, op. cit.

10. Michael Fœssel, « Être reconnu : droit ou fantasme ? », Esprit, $\mathrm{n}^{\circ} 346$, juillet 2008, p. 66.

11. Pierre Bourdieu, Méditations pascaliennes, Paris, Seuil, 1997, p. 119.

12. Gérard Mauger, « vérité objective de l'exploitation et vérité subjective du travail salarié », ContreTemps, $\mathrm{n}^{\circ} 1$, mai 2001, p. 49.

13. P. Bourdieu, op. cit., p. 284.

14. Ibid., p. 200.

15. Bernard Lahire, "Champ, hors-champ, contrechamps », in Bernard Lahire (dir.), Le travail sociologique de Pierre Bourdieu. Dettes et critiques, Paris, La Découverte, 2001, p. 32.

16. Ibid., p. 35.

17. A. Honneth, 2000 op. cit., p. 161.

18. Ibid., p. 165.

19. Estelle Ferrarese, « Pourquoi refuse-t-on la reconnaissance ? Sur les effets de la reconnaissance institutionnelle », in Jean-Paul Payet et Alain Battegay (dir.), La reconnaissance à l'épreuve. Explorations socio-anthropologiques, Villeneuve d'Ascq, Presses universitaires du Septentrion, 2008, p. 97.

20. P. Bourdieu, op. cit., p. 122.

21. Philippe Corcuff, Bourdieu autrement. Fragilités d'un sociologue de combat, Paris, Textuel, p. 111.

22. Ibid., p. 113.

23. A. Honneth, 2000, op. cit., p. 83. 
24. Ibid.

25. Bernard Lahire, L'homme pluriel. Les ressorts de l'action, Paris, Nathan, 1998, p. 204.

26. Georg Herbert Mead, L'esprit, le soi et la société, Paris, PUF, 1963, p. 121.

27. A. Honneth, 2000, op. cit., p. 95.

28. A. Honneth, 2006, op. cit., p. 257.

29. Pierre Bourdieu, Questions de sociologie, Paris, éditions de Minuit, 1984, p. 133.

30. A. Honneth, 2000, op. cit., p. 98.

31. Ibid., p. 106.

32. Ibid., p. 156.

33. Ibid., p. 154.

34. Estelle Ferrarese, "La reconnaissance, le tort et le pouvoir ", in Nancy Fraser, Qu'est-ce que la justice sociale? Reconnaissance et redistribution, Paris, La Découverte, 2005, p. 163.

35. G. H. Mead, 1963, op. cit., p. 121-123.

36. Emmanuel Renault, Mépris social. Éthique et politique de la reconnaissance, Bègles, éditions du Passant, 2004, p. 79-80 et 83.

37. Christian Lazzeri, «Multiple Self et reconnaissance : les processus de construction de l'identité, Le Temps philosophique, $\mathrm{n}^{\circ} 13,2009$, pp. 347.

38. A. Honneth, 2006, op. cit., p. 246.

39. Ibid., p. 248.

40. Ibid., p. 263.

41. Ibid., p. 272.

42. Ibid., p. 311.

43. Ibid., p. 280.

44. Ibid., p. 177.

45. Ibid., p. 248-251-262.

46. P. Bourdieu, 1997, op. cit., p. 225.

47. A. Honneth, 2006, op. cit., p. 97.

48. Ibid., p. 204.

49. Ibid., p. 179.

50. Ibid., p. 212.

51. Ibid., p. 213.

52. Ibid., p. 221.

53. Ibid., p. 218.

54. Ibid., p. 222.

55. Emmanuel Renault, "L'individu comme concept critique», Contretemps, 2009, http://contretemps.eu/interventions/lindividu-comme-concept-critique.

56. Olivier Voirol, «Reconnaissance et méconnaissance : sur la théorie de la violence symbolique ", Information sur les Sciences, vol. 43, n 3, 2004, p. 424.

57. Pierre Bourdieu, Le sens pratique, Paris, éditions de Minuit, 1980, p. 44. 


\section{RÉSUMÉS}

Les débats en sciences et philosophie sociales ayant pour objet le thème de la reconnaissance sont aujourd'hui pléthoriques. Dans le cadre de cet article, nous souhaiterions revenir plus particulièrement sur la théorie de la reconnaissance telle qu'elle est développée par Axel Honneth dans ses plus récents ouvrages, en tant que théorie sociale normative qui a trait à la lutte des sujets pour la reconnaissance mutuelle de leur identité. Ce projet de compréhension critique des sociétés capitalistes avancées a de nombreuses fois fait la preuve de son heuristique, notamment dans le cadre de recherche traitant du travail ou encore de l'exclusion. Nous voudrions toutefois discuter certains de ses attendus théoriques depuis une perspective que l'on pourrait désigner comme un constructivisme de type dispositionnaliste. Ce dialogue permettra d'aller plus loin dans la qualification des phénomènes de reconnaissance, de mépris et d'idéologie. Il nous semble en effet qu'un couplage de cette nature pourrait conduire à améliorer la portée à la fois descriptive et critique du système conceptuel de la théorie de la reconnaissance.

\section{INDEX}

Mots-clés : reconnaissance, mépris, idéologie, champs, dispositions

\section{AUTEUR}

\section{FABIEN GRANJON}

Fabien Granjon est sociologue, professeur en sciences de l'information et de la communication et directeur du Centre d'Études sur les Médias, les Technologies et l'Internationalisation (CEMTI) de l'Université Paris 8 Vincennes à Saint-Denis. 